\title{
RELAÇÕES DA SUTURA CORONÁRIA COM OS SULCOS DA FACE SÚPERO-LATERAL DO LOBO FRONTAL
}

\section{Aplicações neurocirúrgicas}

\author{
Sebastião Gusmão, Cassius Reis, Roberto Leal Silveira, Guilherme Cabral
}

\begin{abstract}
RESUMO - Realizamos estudo anatômico das relações da sutura coronária com os sulcos da convexidade do lobo frontal em sete segmentos cefálicos. Os sulcos pré-central e central distam, respectivamente, $26,5 \mathrm{~mm}$ e $40,5 \mathrm{~mm}$ do bregma e $15,0 \mathrm{~mm}$ e $35,5 \mathrm{~mm}$ do ptério. A utilização da sutura coronária como ponto de referência, e especialmente, sua relação com os sulcos da face súpero-lateral do lobo frontal, permite determinar a projeção sobre o crânio de lesões cerebrais superficiais com a finalidade de delimitar o acesso cirúrgico e orientar a abordagem da convexidade cerebral.
\end{abstract}

PALAVRAS-CHAVE: sutura coronária, sulcos cerebrais, anatomia neurocirúrgica.

\begin{abstract}
Relationships between the coronal suture and the sulci of the lateral convexity of the frontal lobe: neurosurgical applications

ABSTRACT - An anatomical study of the relationships between the coronal suture and the sulci of the convexity of the frontal lobe was accomplished in seven cephalic segments. The pre-central and central sulci are, respectively, $26,5 \mathrm{~mm}$ and $40,5 \mathrm{~mm}$ behind the bregma and $15,0 \mathrm{~mm}$ and $35,5 \mathrm{~mm}$ behind the pterion. The use of the coronal suture as point of reference, and especially, its relationship with the sulci of the lateral surface of the frontal lobe, allows to determine the projection on the cranium of the superficial cerebral lesions with the purpose to delimit the surgical access and to guide the approach to the cerebral convexity.

KEY WORDS: coronal suture, cerebral sulci, neurosurgical anatomy.
\end{abstract}

A topografia cranioencefálica é requisito fundamental para orientar os diferentes acessos à cavidade craniana. O tempo dispendido na cuidadosa delimitação da incisão é altamente recompensador, pois um procedimento cirúrgico simples pode tornar-se difícil e sujeito a complicações pelo posicionamento incorreto da craniotomia. A correta orientação para abordagem neurocirúrgica inicia-se pela identificação de referências anatômicas superficiais. A sutura coronária (StC), em decorrência de sua posição aproximadamente central na abóbada craniana e da fácil identificação, é a referência craniana mais útil nos acessos sobre a convexidade craniana. Ela pode ser localizada na tomografia computadorizada (TC) e na ressonância magnética (RM), palpada in vivo e visibilizada durante a abordagem cirúrgica. Assim, a relação observada em exames de imagem entre a StC e determinada lesão intracraniana pode ser transferida para o paciente. A StC corresponde à junção das bordas posterior do osso frontal e anterior dos ossos parietais. Descreve no centro da abóbada craniana uma linha de concavidade anterior cuja extremidade superior corresponde ao bregma e a inferior ao ptério; seu ponto de união com a linha temporal superior é denominado stefânio. A StC tem relações de proximidade com os sulcos da face súpero-lateral do lobo frontal. Os sulcos horizontais, sulco frontal superior (SFS) e sulco frontal inferior (SFI), cruzam a StC em ângulo aproximadamente reto, enquanto que os sulcos oblíquos, sulco pré-central ( $\mathrm{SPrC}$ ) e sulco central (SC), cursam posterior e paralelamente à StC. O conhecimento dessas relações e das distâncias entre a StC e esses sulcos permite ao cirurgião orientar-se durante o acesso cirúrgico.

Em 1861, Broca' estudou a relação entre a StC e o SC. Por meio de consulta ao Index Medicus para publicações anteriores a 1966 e da Medline no perío-

Laboratório de Microcirurgia da Faculdade de Medicina da Universidade Federal de Minas Gerais (UFMG), Belo Horizonte MG, Brasil.

Recebido 12 Fevereiro 2001. Aceito 18 Abril 2001.

Dr. Sebastião Gusmão - Rua Padre Rolim 921/21 - 30130-090 Belo Horizonte MG - Brasil. FAX: 0312248452.

E-mail:gusmao@medicina.ufmg.br 


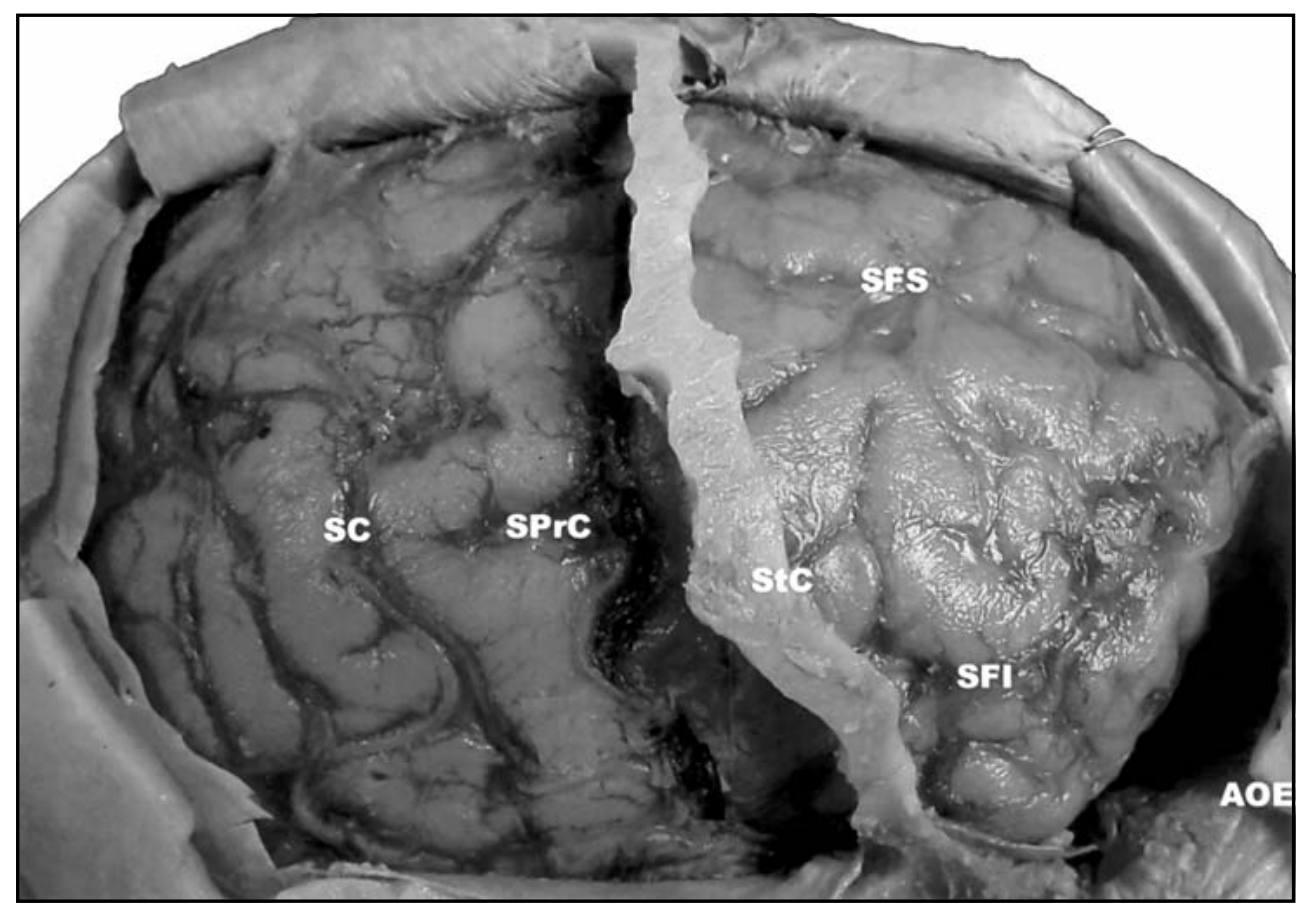

Fig 1. Craniotomia fronto-parietal deixando ponte óssea correspondente às bordas posterior do osso frontal e anterior do osso parietal (sutura coronária). Observam-se as relaçôes da sutura coronária (StC) com a apófise orbitária externa (AOE) e os sulcos frontal superior (SFS), frontal inferior (SFI), pré-central (SPrC) e central (SC).

do de 1966 a 2000, encontrou-se apenas um artigo sobre a StC que estuda sua relação com o giro frontal inferior e as implicações na leucotomia pré-frontal ${ }^{2}$.

O objetivo do presente estudo é determinar as relações da StC com os sulcos (e indiretamente com os giros) da face súpero-lateral (convexidade) do lobo frontal.

\section{MÉTODOS}

Sete segmentos cefálicos de cadáveres humanos, submetidos à fixação em solução de formol e vasos injetados com silicone colorido, foram utilizados para o estudo das relações entre a StC e os sulcos da convexidade do lobo frontal.

Realizou-se craniotomia fronto-parietal bilateral com preservação de ponte óssea correspondente à StC (Fig 1). A dura-máter foi aberta e pediculada junto ao seio sagital superior. Seguiu-se identificação, por meio da dissecção da aracnóide, com o auxílio do microscópio cirúrgico, dos sulcos da convexidade do lobo frontal.

Foram medidas as seguintes distâncias: StC (bregma) - násio; StC (ptério) - apófise orbitária externa (AOE); StC (bregma) - SPrC; StC (bregma) - SC; StC (ptério) - SPrC; StC (ptério) - SC; Bregma - interseção StC/SFS; Stefânio interseção StC/SFI (Fig 2).

\section{RESULTADOS}

O bregma (extremidade superior da StC) encontra-se, em média, a 133,5 mm $( \pm 3,7)$ do násio e o ptério (extremidade inferior da StC) a $33,6 \mathrm{~mm}$ $( \pm 3,7)$ da apófise orbitária externa. O SPrC e o SC distam, respectivamente, $26,5 \mathrm{~mm}( \pm 6,7)$ e $40,5 \mathrm{~mm}$ $( \pm 7,9)$ do bregma e $15,0 \mathrm{~mm}( \pm 7,4)$ e $35,5 \mathrm{~mm}$ $( \pm 7,4)$ do ptério. O ponto de interseção StC/SFS dista $38,5 \mathrm{~mm}( \pm 6,9)$ do bregma e o ponto de interseção StC/SFI encontra-se $9,0 \mathrm{~mm}( \pm 5,7)$ abaixo do stefânio (Tabela 1, Fig 2).

\section{DISCUSSÃO}

De acordo com os dados obtidos, a StC inicia-se na linha médio-sagital (bregma) a 133,5 mm do násio; descreve uma linha de concavidade anterior, terminando no ptério, a 33,6 mm da apófise orbitária externa (processo zigomático do osso frontal). Seeger $^{3}$ encontrou o valor de $130 \mathrm{~mm}$ para a distância násio-bregma. A $38,5 \mathrm{~mm}$ do bregma, a StC passa sobre o SFS, e a 9,0 $\mathrm{mm}$ abaixo do stefânio ela cruza o SFI.

Tanto os sulcos pré-central e central como a StC apresentam trajeto de direção oblíqua nos sentidos súpero-inferior e póstero-anterior. Como a inclinação dos sulcos é maior que a inclinação da StC, eles aproximam-se progressivamente desta sutura à medida que descrevem trajeto descendente (Figs 1 e 2). Assim, as distâncias do SPrC às extremidades superior (bregma) e inferior (ptério) da StC são, res- 
Tabela 1. Medida das distâncias (em mm) entre a sutura coronária e referências cranianas (bregma; apófise orbitária externa: AOE) e sulcos da face súpero-lateral do lobo frontal (sulco frontal superior: SFS; sulco frontal inferior: SFI; sulco pré-central: SPrC; sulco central: SC) nos lados direito (D) e esquerdo (E). Média (Med), mediana (Medn) e desvio-padrão (Des).

\begin{tabular}{|c|c|c|c|c|c|c|c|c|c|c|c|c|c|c|c|c|c|}
\hline \multicolumn{18}{|c|}{ Peças anatômicas } \\
\hline Distâncias & \multicolumn{2}{|c|}{1} & \multicolumn{2}{|c|}{2} & \multicolumn{2}{|c|}{3} & \multicolumn{2}{|c|}{4} & \multicolumn{2}{|c|}{5} & \multicolumn{2}{|c|}{6} & \multicolumn{2}{|c|}{7} & Med & Medn & Des \\
\hline Lado & $\mathrm{D}$ & $E$ & $\mathrm{D}$ & E & $D$ & $\mathrm{E}$ & $\mathrm{D}$ & $E$ & $\mathrm{D}$ & $\mathrm{E}$ & $\mathrm{D}$ & $E$ & $D$ & $E$ & & & \\
\hline Bregma-násio & \multicolumn{2}{|c|}{130} & \multicolumn{2}{|c|}{135} & \multicolumn{2}{|c|}{135} & \multicolumn{2}{|c|}{130} & \multicolumn{2}{|c|}{140} & \multicolumn{2}{|c|}{135} & \multicolumn{2}{|c|}{130} & 133,5 & 135,0 & 3,7 \\
\hline Bregma - SPrC & 32 & 40 & 30 & 31 & 27 & 23 & 25 & 25 & 25 & 27 & 30 & 28 & 15 & 13 & 26,5 & 27,0 & 6,7 \\
\hline Bregma - SC & 55 & 50 & 40 & 45 & 36 & 43 & 45 & 41 & 37 & 35 & 42 & 45 & 30 & 24 & 40,5 & 41,5 & 7,9 \\
\hline Bregma-StC/SFS & 34 & 44 & 37 & 35 & 43 & 35 & 50 & 30 & 50 & 45 & 35 & 40 & 31 & 30 & 38,5 & 36,0 & 6,9 \\
\hline Ptério - AOE & 32 & 34 & 28 & 31 & 32 & 36 & 35 & 33 & 40 & 34 & 30 & 37 & 29 & 40 & 33,6 & 33,5 & 3,7 \\
\hline Ptério - SPrC & 19 & 20 & 11 & 8 & 9 & 6 & 8 & 33 & 25 & 18 & 16 & 15 & 12 & 11 & 15,0 & 13,5 & 7,4 \\
\hline Ptério - SC & 35 & 31 & 40 & 38 & 20 & 20 & 32 & 46 & 33 & 32 & 24 & 35 & 31 & 25 & 35,5 & 32,0 & 7,4 \\
\hline Stefânio-StC/SFI & 16 & 9 & 14 & 0 & 10 & 10 & 7 & 5 & 19 & 15 & 3 & 0 & 8 & 10 & 9,0 & 9,5 & 5,7 \\
\hline
\end{tabular}

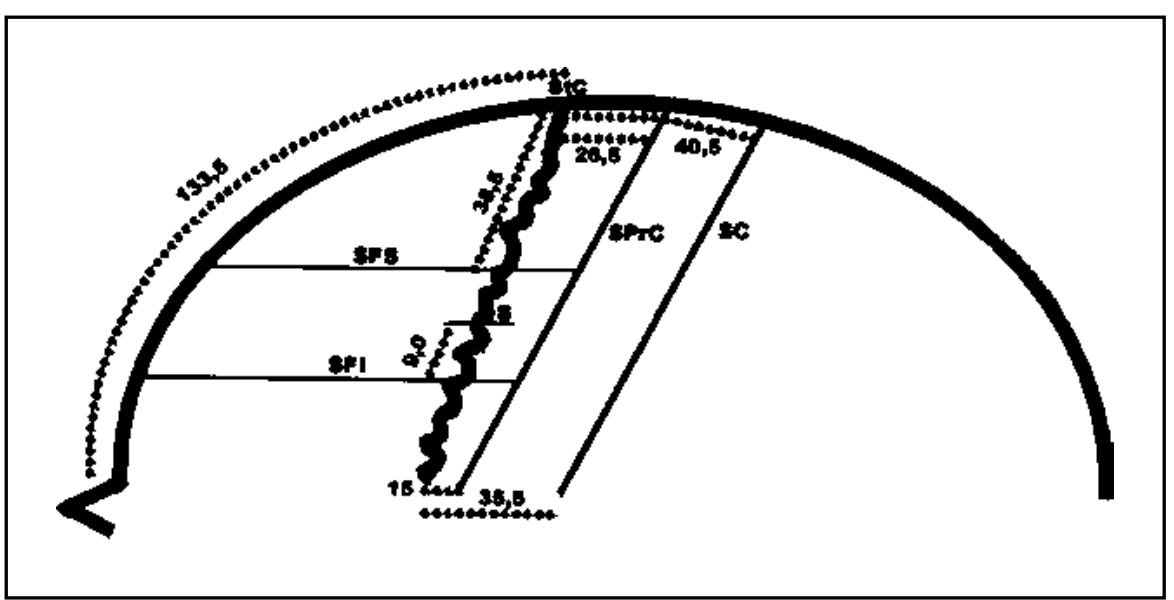

Fig 2. Esquema mostrando as relações e as distâncias entre a sutura coronária (StC) e os sulcos da face súpero-lateral do lobo frontal. SFS: sulco frontal superior; SFI: sulco frontal inferior; SPrC: sulco pré-central; SC: sulco central; S: stefânio. pectivamente, de $26,5 \mathrm{~mm}$ e $15,0 \mathrm{~mm}$, enquanto que estas distâncias em relação ao SC são, respectivamente, de 40,5 mm e 35,5 mm. Estes dados estão próximos dos descritos na literatura. Broca ${ }^{4}$ encontrou os seguintes valores para as distâncias entre os sulcos pré-central e central e as extremidades superior (bregma) e inferior (ptério) da StC: bregma-extremidade superior do $\mathrm{SPrC}=28 \mathrm{~mm}$, ptério-extremidade inferior da $\mathrm{StC}=10 \mathrm{~mm}$, bregma- extremidade superior do $\mathrm{SC}=47 \mathrm{~mm}$ e ptério-extremidade inferior do SC $=28 \mathrm{~mm}$. Rowland e col. ${ }^{2}$ também encontraram o valor de $28 \mathrm{~mm}$ para a distância ptério-extremidade inferior do SC e concluiram que o terço inferior da StC corresponde à parte triangular do giro frontal inferior e encontra-se à frente do SPrC.

Apesar do presente estudo basear-se em pequena amostragem, verificaram-se valores estatisticamente confiáveis em decorrência dos pequenos valores de desvio-padrão obtidos.
Na TC, a sutura coronária aparece como entalhe na tábua interna, determinado pela transição dos ossos frontal e parietal, e apresenta maior atenuação (densidade) em relação ao restante do osso. $\mathrm{Na}$ $\mathrm{RM}$, tanto nas sequências ponderadas em T1 como em T2, o osso compacto (cortical) aparece em cor preta e a díploe em branco. Assim, nos cortes da RM, o osso aparece como uma faixa escura (tábuas externa e interna) com uma linha branca no meio (díploe); na sutura coronária esta linha branca da díploe é interrompida (Fig 3).

A StC pode ser traçada sobre o couro cabeludo. Sua extremidade superior (bregma) pode ser identificada pela palpação ou localizada a aproximadamente $133,5 \mathrm{~mm}$ atrás do násio. Sua extremidade inferior (ptério) encontra-se aproximadamente a 33,6 $\mathrm{mm}$ da apófise orbitária externa.

Determinado ponto da convexidade cerebral pode ser transferido da TC ou da RM para a superfície 


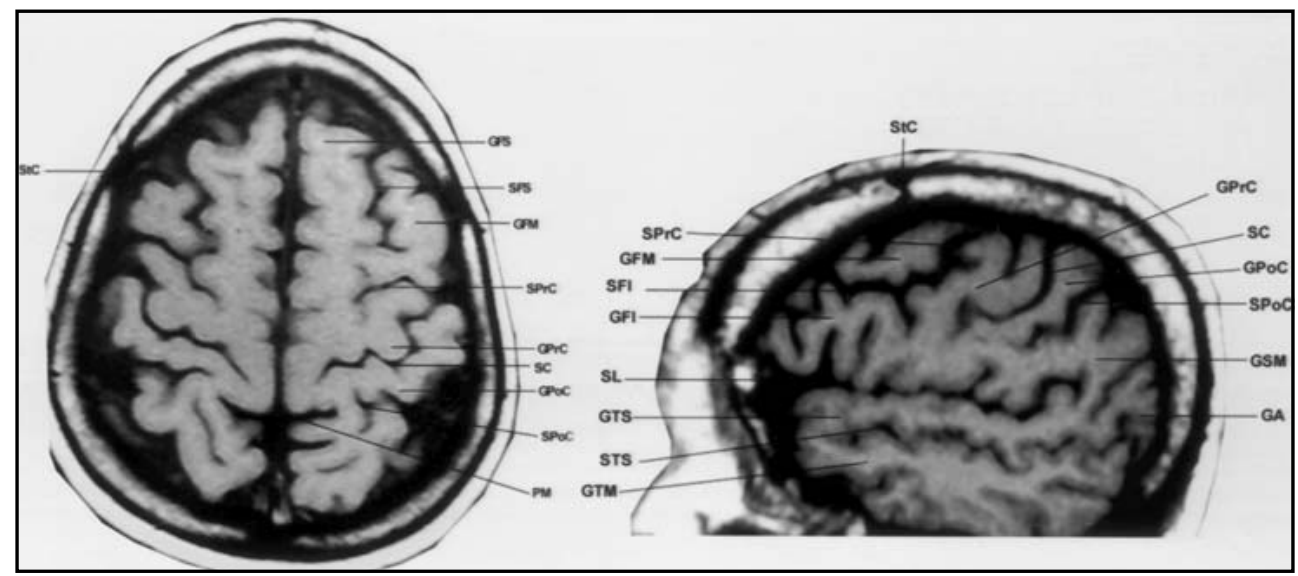

Fig 3. Cortes axial (esquerda) e sagital (direita) da ressonância magnética mostrando as relações da sutura coronária (StC) com os sulcos da face súpero-lateral do lobo frontal. SFS: sulco frontal superior, SFI: sulco frontal inferior, SPrC: sulco pré-central, SC: sulco central, SPOC: sulco pós-central, PM: pars marginalis do giro do cíngulo, GFS: giro frontal superior, GFM: giro frontal médio, GFI: giro frontal inferior, GPrC: giro pré-central, GPoC: giro pós-central, GSM: giro supramarginal, GA: giro angular, SL: sulco lateral, STS: sulco temporal superior, GTS: giro temporal superior, GTM: giro temporal médio.

Fig 4. A interseção quase em ângulo reto entre a sutura coronária e a linha mediosagital cria um sistema de coordenadas cartesianas sobre a superfície craniana. Este sistema permite a localização de um ponto sobre o crânio por meio do conhecimento das distâncias entre o ponto e as linhas formadas pela sutura coronária e pela linha mediosagital.

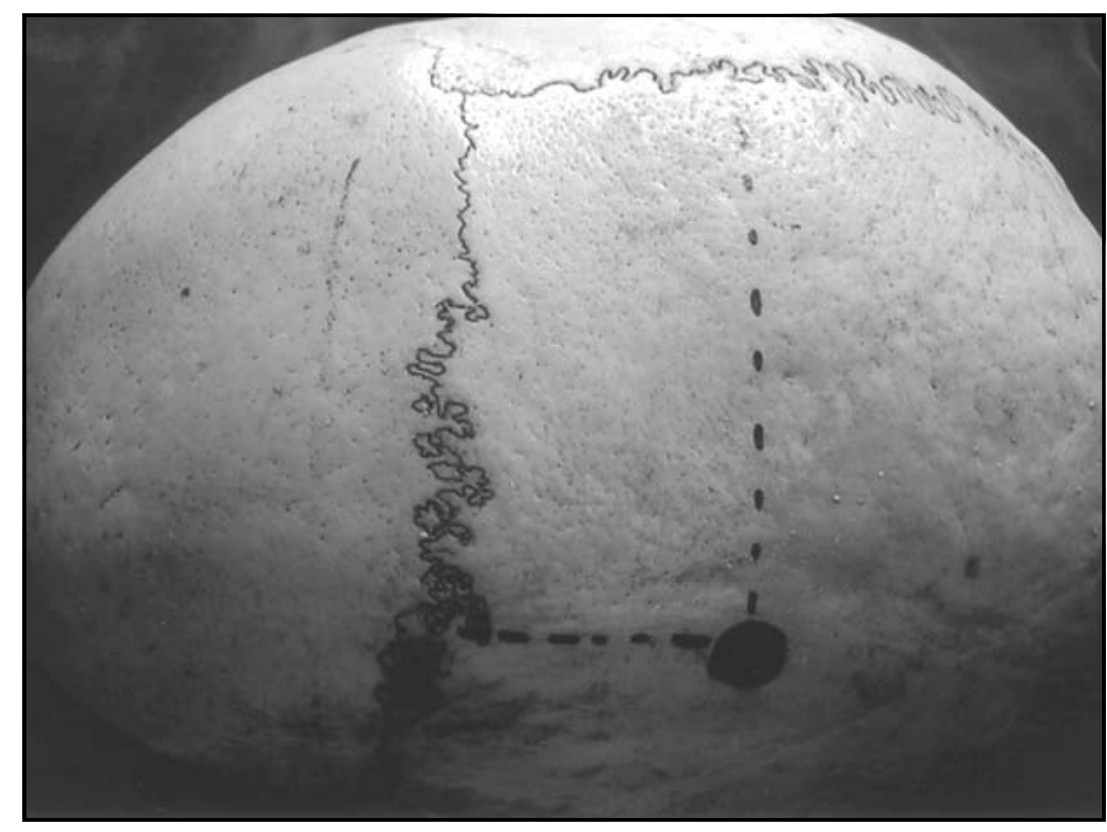

craniana do paciente, usando-se como referência a StC, que pode ser identificada nos exames de imagem (TC e RM) e traçada sobre a cabeça do paciente. O ponto da lesão intracraniana deve ser transferido para a cabeça do paciente no sentido ânteroposterior ou sagital (distância entre a lesão e a StC) e súpero-inferior ou coronal (distância entre a lesão e a linha médio-sagital). As medidas das distâncias no exame de imagem são realizadas usando-se a régua impressa no próprio exame.

A interseção quase em ângulo reto entre a StC e a linha médio-sagital cria um sistema de coordena- das cartesianas sobre a superfície craniana. Este sistema permite a localização de um ponto sobre o crânio por meio do conhecimento das distâncias entre o ponto e as linhas formadas pela StC e pela linha médio-sagital (Fig 4).

A localização no sentido ântero-posterior é feita determinando-se a distância entre o ponto da lesão e a StC, medida nos cortes axiais da TC ou da RM. Quando a lesão é próxima da linha média (paramediana ou parasagital), pode-se também medir a distância entre o ponto da lesão e o bregma no corte sagital paramediano da RM. 


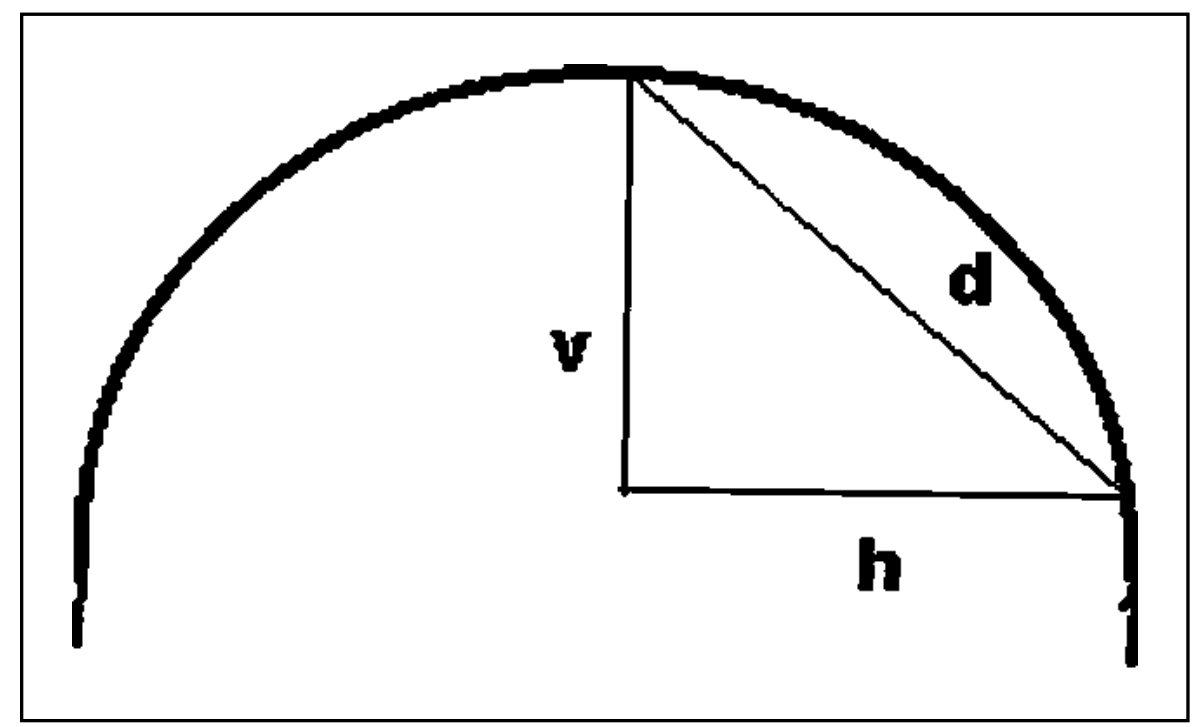

Fig 5. Esquema correspondente ao corte coronal da ressonância magnética para localização de lesão intracraniana lateral. O comprimento da linha horizontal que vai da projeção da lesão sobre a parede craniana à linha média (h) e o comprimento da linha vertical que vai da projeção da lesão na linha média à linha mediosagital (v) representam os catetos de um triângulo retângulo. Usando-se o teorema de Pitágoras, calcula-se a hipotenusa, que representa a distância, em linha reta, da lesão à linha mediosagital: $d=\sqrt{h^{2}+v^{2}}$. Quando transferida para o crânio do paciente esta distância é alguns milímetros menor que a real devido à convexidade da abóbada craniana. Os valores de h e v podem também ser obtidos no corte axial e no topograma da tomografia computadorizada (ver Fig 6).

Para demarcar o ponto da lesão no sentido supero-inferior nas lesões paramedianas, mede-se a distância entre a lesão e a linha mediosagital (corte coronal obtido por RM) ou a distância entre a linha de corte da lesão e a linha mediosagital (topograma da TC). Esta medida é tanto mais precisa quanto menor for a distância.

Nas lesões laterais, a distância entre o ponto de projeção da lesão no crânio e a linha média, medida na TC ou na RM, será significativamente menor que a distância entre a linha mediosagital e o ponto de projeção da lesão na parede lateral do crânio (medido sobre a cabeça) em razão da convexidade da abóbada craniana. Utilizando-se o teorema de Pitágoras, pode-se conseguir correspondência mais precisa da distância entre a lesão e a linha média (medida no exame de imagem) e a distância da lesão à linha mediosagital (medida sobre o crânio).

Mede-se, na imagem tomográfica ou nos cortes axial e coronal da RM, o comprimento da linha que liga a parede craniana à linha mediana e que passa pelo centro da lesão. A seguir, mede-se a distância da projeção da lesão na linha média (corte coronal da RM) ou da linha de corte da lesão (topograma) à linha mediosagital. Estas duas medidas representam os catetos de um triângulo retângulo. Por meio do teorema de Pitágoras, calcula-se a hipotenusa, que representa a distância, em linha reta, do ponto de projeção da lesão na parede craniana à linha mediosagital. Assim, a distância, sobre a convexidade craniana, do ponto de projeção da lesão na parede craniana à linha mediosagital é dada pela equação $d=\sqrt{h^{2}+v^{2}}$, onde d é a distância, em linha reta, da lesão à linha mediosagital, h o comprimento da linha horizontal que vai da projeção da lesão sobre a parede craniana à linha média e $v$ o comprimento da linha vertical que vai da projeção da lesão na linha média à linha mediosagital. Quando transferida para o crânio do paciente, esta distância é alguns milímetros menor que a real devido à convexidade da abóbada craniana (Fig 5).

Os valores assim obtidos têm-se mostrado bastante precisos em nossa prática de representar sobre a convexidade craniana a projeção de lesões corticais com a finalidade de delinear a craniotomia centrada sobre a lesão. A precisão do processo pode ser também demonstrada pela comprovação prática que realizamos utilizando uma calota craniana. Colocamos neste modelo um artefato sob a tábua óssea interna (simulando uma lesão cortical) em um ponto distando $70 \mathrm{~mm}$ da sutura sagital (medido sobre a convexidade craniana) e $30 \mathrm{~mm}$, posterior- 


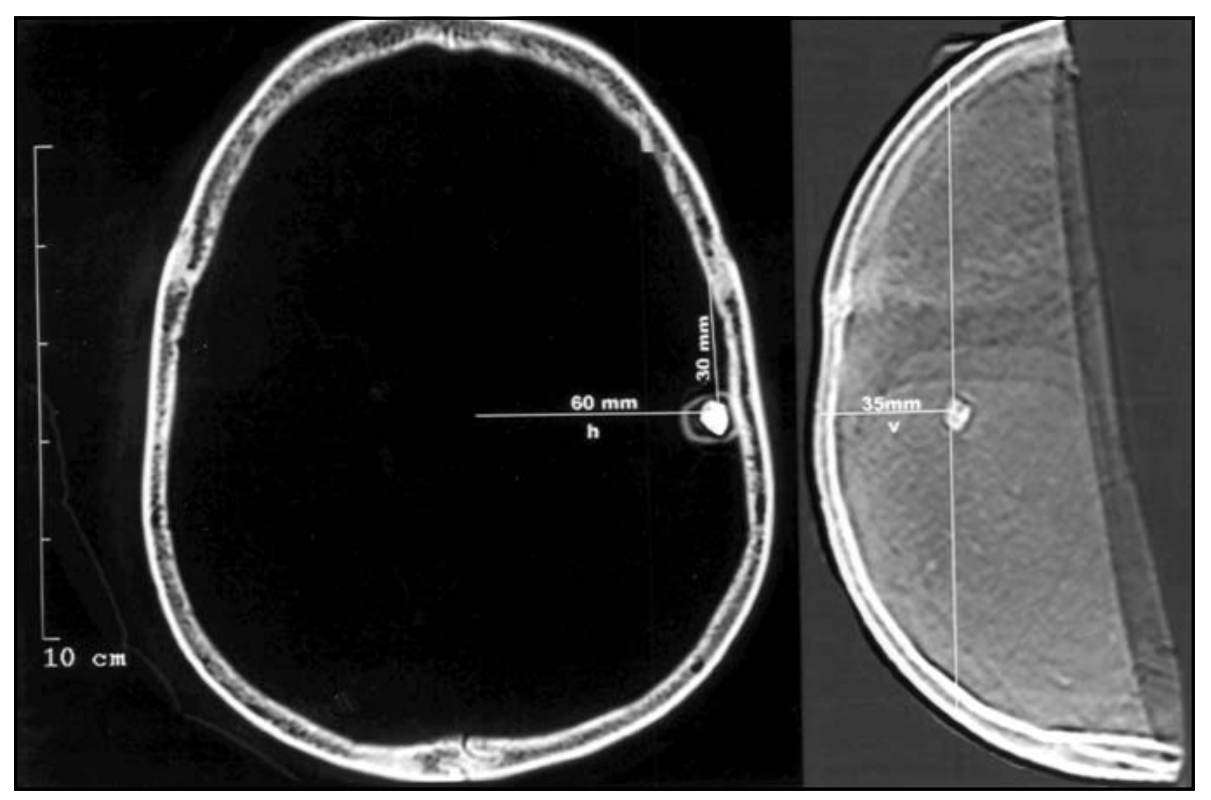

Fig 6. À esquerda, corte tomográfico mostrando as distâncias do ponto de projeção do artefato na convexidade craniana à StC e à linha média (h); à direita, topograma mostrando a distância do plano de corte à linha mediosagital (v).

mente, da StC (Fig 4). A distância do ponto de projeção deste artefato na convexidade craniana à linha média (h) era $60 \mathrm{~mm}$ e a distância de sua projeção sobre a linha média à sutura sagital (v) era $35 \mathrm{~mm}$. Usando-se a equação $d=\sqrt{h^{2}+v^{2}}$, obtém-se o valor de $69 \mathrm{~mm}$, bastante próximo da distância real medida sobre a convexidade craniana do ponto de projeção da lesão sobre o crânio à sutura sagital (70 $\mathrm{mm}$ ).

Este mesmo modelo foi submetido a TC, obtendo-se os valores de $31 \mathrm{~mm}$ para a distância da lesão à StC, $61 \mathrm{~mm}$ para a distância do ponto de projeção do artefato na convexidade craniana à linha média (h) e de $33 \mathrm{~mm}$ para a distância do ponto de projeção do artefato sobre a linha média à sutura sagital (v) (medido no topograma) (Fig 6). Usando-se a equação $d=\sqrt{h^{2}+v^{2}}$, obtém-se o valor de $69 \mathrm{~mm}$, bastante próximo da distância, medida sobre a convexidade craniana, do ponto de projeção da lesão sobre o crânio à sutura sagital $(70 \mathrm{~mm})$.

Tendo-se a distância da lesão em relação à StC (30 $\mathrm{mm}$ posterior) e á linha médiosagital $(70 \mathrm{~mm}$ abaixo), pode-se inferir a localização cerebral da mesma. Como a StC tem um comprimento de aproximadamente $100 \mathrm{~mm}$, uma lesão em sua porção inferior e $30 \mathrm{~mm}$ posterior deve estar próxima do SC, pois a extremidade inferior deste sulco encontra-se, em média, a 35,5 mm da StC. Tal indução poderá ser confrontada com o exame de imagem.

Os sulcos da convexidade cerebral podem ser identificados com precisão na TC e na $\mathrm{RM}^{6,7}$. O SFS aparece nos cortes axiais da TC e da RM como uma linha paralela e, aproximadamente, a $21 \mathrm{~mm}$ da fissura inter-hemisférica, terminando em ângulo reto no SPrC. O SFI é identificado nos cortes sagitais laterais da RM como uma linha que termina no ramo inferior do $\operatorname{SPrC}^{6,7}$ (Fig 3).

O SPrC aparece nos cortes axiais altos da TC e da RM como linha oblíqua dirigida no sentido pósteroanterior e da linha média para o entalhe da sutura coronária, aproximadamente $26 \mathrm{~mm}$ posterior a esta sutura. Nestes mesmos cortes, o SC apresenta conformação em gancho ou C (joelho médio, hook sign) com concavidade anterior apontando para o SFS e separa os giros pós-central (mais estreito) e pré-central; sua extremidade superior projeta-se como uma linha oblíqua na pars marginalis do giro do cíngulo (bracket sign) a $47 \mathrm{~mm}$ da StC. A extremidade inferior do SC é identificada nos cortes sagitais laterais da RM pela fusão dos giros pré e pós-central para formar o giro subcentral, de concavidade superior ${ }^{6,7}$ (Fig 3).

A relação entre os sulcos frontais e a lesão, definida nos exames de imagem, permitirá maior precisão à localização sobre a convexidade cerebral. Identificando-se os sulcos frontais e a StC e transferindo-se as relações entre os mesmos para a superfície craniana (topografia cranioencefálica), poderá ser localizada a lesão tanto em relação à StC como em relação aos sulcos, permitindo delineamento mais preciso do acesso e melhor orientação durante a abordagem cirúrgica.

Broca $^{4}$ foi o primeiro, em 1871, a realizar craniotomia orientada pela correlação de localização cerebral à topografia cranioencefálica, sendo esta a primeira vez que a localização de lesão intracraniana 
não identificada visualmente foi seguida de intervenção neurocirúrgica. O paciente apresentava quadro de afasia expressiva, indicando lesão da parte opercular do giro frontal inferior esquerdo. Dez anos antes, o próprio Broca havia identificado o centro de expressão da linguagem neste giro (área de Broca) e realizado os trabalhos pioneiros de topografia cranioencefálica ${ }^{1}$. Ele determinou que as extremidades inferiores da StC e do SPrC distavam, aproximadamente, $10 \mathrm{~mm}$ e que entre ambos encontrava-se a parte opercular do giro frontal inferior (área de Broca; área 44 de Brodmann). Através de orifício de trefina centrado imediatamente atrás da extremidade inferior da StC foi retirado um abscesso extradural.

A utilização da StC como ponto de referência, e especialmente, sua relação com os sulcos da face súpero-lateral do lobo frontal, permitem determi- nar a projeção sobre o crânio de lesões cerebrais superficiais com a finalidade de delimitar o acesso cirúrgico e orientar a abordagem da convexidade cerebral.

\section{REFERÊNCIAS}

1. Broca P. Sur les rapports anatomiques des divers points de la surface du crâne e des diverses parties des hémisphères cérébraux. Bull Soc d'Anth 1861;2:340-348.

2. Rowland LP, Mettler FA. Relation between the coronal suture and cerebrum. J Comp Neurol 1948;89:21-40.

3. Seeger $W$. The microsurgical approaches to the target areas of the brain. Wien: Springer-Verlag, 1993:73.

4. Broca P. Sur la topographie cranio-cérébrale ou sur les rapportes anatomiques du crane et du cerveau. Rev d'Anthrop 1876;5:193-248.

5. Testut L, Jacob O. Traité d'anatomie topographique. Paris: Gaston Doin Editeurs, 1929:1201.

6. Kido DK, LeMay M, Levinson AW, Benson WE. Computed tomographic localization of the precentral gyrus. Radiology 1980;135:373-377.

7. Naidich TP, Brightbill TC. Systems for localizing fronto-parietal gyri and sulci on axial CT and MRI. Int J Neuroradiol 1996;2:313-338. 